\title{
EP-83
}

\section{Diaphragmatic hernia following living-donor right hepatectomy: Experience of a high-volume transplant center}

\author{
Hui-Ju Kim, Dong-Hwan JUNG*, Young-In YOON
}

Division of Hepatobiliary Surgery and Liver Transplantation, Asan Medical Center, University of Ulsan College of Medicine, Seoul, Korea

Introduction: Right diaphragmatic hernia (DH) occurs more in recipients or those receiving hepatectomy (for neoplasms, not for transplantation), but very rarely in donor patients.

Methods: Between November 1994 and December 2020, we experienced DH following living-donor right hepatectomy in 6 of 5,672 adult LDLT. Here, we report successful treatment of DH and evaluate long-term outcomes by reviewing the medical records of the 6 patients.

Results: All 6 patients presented with abdominal pain and underwent diaphragmatic repair at a median time interval of 4.95 years (range, 0.24-17.00 years) after hepatectomy. All patients were diagnosed by computerized tomography scan. Except for one patient who underwent an abdominal approach, the rest of the patients had a right thoracotomy with lysis of the adhesions, reduction of the hernia, and primary repair of the diaphragm. At last follow-up, all 6 patients remain normal chest X-ray examination demonstrating no recurrence of diaphragmatic hernia, and normal liver functions tests.

Conclusions: Although rare, diaphragmatic hernia should be considered as an important potential late complication in live donor underwent right hepatectomy. The screening protocol for early identification should be applied to prevent the development of complications. 